\title{
Intravenous immunoglobulin treatment in lower motor neuron disease associated with highly raised anti-GM1 antibodies
}

\author{
L H van den Berg, H Franssen, P A Van Doorn, J H J Wokke
}

\begin{abstract}
The effect of intravenous immunoglobulin (IVIg) treatment was studied in five patients with lower motor neuron disease associated with highly raised anti-GM1 antibodies but without evidence of conduction block on neurophysiological examination. The patients received IVIg treatment $(0.4 \mathrm{~g} / \mathrm{kg}$ for five consecutive days) in an open study. Only one patient responded to IVIg treatment, which was confirmed in a double blind, placebo controlled study (two placebo treatments and two IVIg treatments in a randomised order). However, after six months of maintenance IVIg treatment $(0.4 \mathrm{~g} / \mathrm{kg}$ weekly $)$ muscle weakness gradually deteriorated below pretreatment levels despite continued treatment. It is concluded that the presence of raised anti-GM1 antibodies does not identify a subgroup of patients with lower motor neuron disease who respond to IVIg treatment and although some patients with lower motor neuron disease may initially respond, IVIg treatment does not seem to be sufficient as long term treatment.
\end{abstract}

(F Neurol Neurosurg Psychiatry 1997;63:674-677)

Keywords: lower motor neuron disease; anti-GM1 antibodies; intravenous immunoglobulin treatment

The pathogenesis of motor neuron disease is unknown. A role for autoimmunity has been suggested, but all treatment strategies in this direction have proved rather disappointing. ${ }^{1}$ The identification of multifocal motor neuropathy by the electrophysiological demonstration of motor conduction block in patients presenting with manifestations of a pure lower motor neuron disease has been important as multifocal motor neuropathy is a potentially treatable disease. ${ }^{2-9}$ Multifocal motor neuropathy is often associated with raised antiGM1 antibodies but raised titres have also been found in Guillain-Barré syndrome and in lower motor neuron disease without conduction block. ${ }^{3-12}$ Despite many reports on the clinical correlation of anti-GM1 antibody titres in neurological disease, little is known about its predictive value for identification of a treatable disease. Improvement of muscle strength after cyclophosphamide treatment has been reported in patients with multifocal motor neuropathy, as well as in patients with lower motor neuron disease associated with raised anti-GM1 antibodies. ${ }^{51013}$ More recently, several studies have shown a beneficial effect of intravenous immunoglobulin (IVIg) treatment in patients with multifocal motor neuropathy..$^{6-9}$ We investigated whether a similar response can be found in patients with lower motor neuron disease associated with raised anti-GM1 antibodies but without conduction block. In the patient who improved the beneficial effect of IVIg was confirmed in a single patient double blind, placebo controlled trial and subsequently the effect of long-term IVIg maintenance treatment was measured.

\section{Patients and methods}

PATIENTS

From 45 patients with lower motor neuron disease five (one woman, four men; age range 16 to 62) were selected on the basis of the presence of highly raised serum anti-GM1 antibodies and the absence of conduction block on electrophysiological examination. All patients had a predominantly distal asymmetric limb weakness with gradual progression for two to six years. Reflexes were low or absent in affected limbs. None of the patients had upper motor neuron or bulbar features.

\section{ELECTRODIAGNOSTIC STUDIES}

In all patients electrophysiological findings were compatible with lower motor neuron disease: spontaneous muscle fibre activity or signs of reinnervation on EMG, decreased amplitude of compound muscle action potential (CMAP), normal or near normal motor conduction velocity, and normal sensory conduction. Sensory conduction was normal across common entrapment sites in the arm.

To detect conduction block or features of demyelination the following investigations were done. Motor conduction was measured at both sides in the following nerves: median (to the abductor pollicis brevis and the flexor 
carpi radialis muscles), ulnar (abductor digiti minimi), radial (extensor carpi ulnaris), musculocutaneus (biceps brachii), deep peroneal (extensor digitorum brevis), and tibial (abductor hallucis brevis), using surface electrodes. Stimulation sites were the wrist, $5 \mathrm{~cm}$ below and $5 \mathrm{~cm}$ above the elbow, axilla, Erb's point, ankle, and $5 \mathrm{~cm}$ below and $5 \mathrm{~cm}$ above the fibular head and popliteal fossa. When the median and ulnar nerve were stimulated at the axilla or Erb's point, a collision technique was used to avoid contamination of the CMAP due to stimulus spread to another nerve. ${ }^{14}$ When stimulation at Erb's point disclosed abnormalities, the stimulator was set at maximal output. F waves were recorded after 20 stimuli delivered to the median, ulnar, deep peroneal, and tibial nerves at the wrist or ankle. Before the conduction studies the limbs were warmed in water at $37^{\circ} \mathrm{C}$ for 30 minutes. ${ }^{15}$ For each nerve segment the amplitude, area, and duration of the negative part of the CMAP on proximal stimulation was compared with that on distal stimulation to detect definite conduction block (area reduction of at least $50 \%$ ), ${ }^{16}$ possible conduction block (amplitude reduction of at least $30 \%$ for arm nerves and $40 \%$ for leg nerves), ${ }^{17}$ or increased temporal dispersion (duration increase of at least $30 \%$ ). ${ }^{18}$ Conduction block was only considered when the CMAP amplitude on distal stimulation exceeded $1.0 \mathrm{mV}$. Evidence of demyelination included: (1) conduction block, (2) increased temporal dispersion, (3) reduction of motor conduction velocity, or increased distal motor latency, or increased minimal $\mathrm{F}$ wave latency according to previously described criteria for demyelination, ${ }^{19}$ (4) absent $\mathrm{F}$ waves.

ANTI-GM1 ANTIBODIES

IgM and IgG anti-GM1 antibodies were measured before and after treatment as described previously. ${ }^{11}$ The upper limit of normal (200 AU/l) was set at the highest titre obtained from 50 normal and 192 disease controls. ${ }^{11}$ Raised anti-GM1 antibodies were of the IgM isotype and ranged from 1450 to $5300 \mathrm{AU} / \mathrm{l}$; anti-GM1 antibodies of the IgG isotype were not raised in any of 45 patients with lower motor neuron disease.

IVIg TREATMENT PROTOCOL

The IVIg treatment protocol included an open trial, a single patient double blind placebo controlled designed trial, and long term maintenance IVIg treatment. ${ }^{7}$

Open trial

Patients were treated with IVIg (Central Laboratory Blood Transfusion, Amsterdam; $0.4 \mathrm{~g} / \mathrm{kg}$ for five consecutive days). The patient who improved as defined later entered the double blind placebo controlled trial, which was started when the patient had returned clinically to the pretreatment state.

Double blind placebo controlled trial

The patient received two IVIg treatments $(0.4$ $\mathrm{g} / \mathrm{kg}$ for five consecutive days) and two placebo treatments (pasteurised plasma solution for five consecutive days) in a randomised order. Treatments were blinded for the patient and the physician by the pharmacist. To prevent cumulative dose effects, the interval between each treatment was determined by the time it took the patient to return clinically to the pretreatment state, and the minimal time between two treatment courses was kept at one month. The code was disclosed by the participating pharmacist after all treatment courses had been completed and all measurements evaluated.

\section{Assessment of treatment response}

To ensure similar conditions for each treatment, the patient was admitted to hospital for six consecutive days during each treatment course. The patient was examined before and after each treatment (days 1 and 6 of admission) and then weekly at the outpatient clinic by the same physician (LHvdB). Muscle strength (flexors and extensors of the neck, elbow, wrist, hip, knees, and feet; abductors of the upper arm; hand grip; and abductor and opponens of the thumb) was evaluated with a hand held dynamometer and the Medical Research Council (MRC) scale. ${ }^{7}$ We used the dynamometric measurements to define improvement of muscle strength: an increase of $50 \%$ or more had to be present in at least two muscles or muscle groups, without a decrease of at least $25 \%$ in more than one muscle or muscle group. To study the correlation between these measurements and subjective improvement of the patient, the patient was asked to state the order in which he thought that he had received IVIg or placebo at the end of all treatment courses.

Long term maintenance treatment

The patient who responded to IVIg but not to placebo subsequently received a full course of IVIg treatment $(0.4 \mathrm{~g} / \mathrm{kg}$ for five days) followed by maintenance IVIg (one IVIg infusion (0.4 $\mathrm{g} / \mathrm{kg}$ ) every week) which was previously shown to be effective as maintenance treatment in multifocal motor neuropathy. ${ }^{8}$

\section{Results}

\section{PATIENTS}

In the open study only one patient improved after IVIg treatment. This 47 year old man had an asymmetric weakness of both arms and legs that had developed over five years. The initial symptom was weakness of the right intrinsic hand muscles. Physical examination at onset of treatment disclosed mild weakness (MRC 4) of the upper arm abductors (right $(\mathrm{R})>$ left $(\mathrm{L})$ ), elbow flexors $(\mathrm{L}>\mathrm{R})$ and extensors $(\mathrm{L}=\mathrm{R})$, hip flexors $(\mathrm{R}>\mathrm{L})$, knee flexors $(\mathrm{R}>\mathrm{L})$ and foot flexors $(R>L)$, and extensors $(R>L)$, and severe weakness (MRC 2-3) of the wrist flexors and extensors and intrinsic hand muscles $(\mathrm{L}=\mathrm{R})$. All deep tendon reflexes were absent. Weakness was associated with muscle wasting in most muscle groups. There were no upper motor neuron or bulbar features and sensation was intact. The protein content of CSF was normal $(0.47 \mathrm{~g} / \mathrm{l})$. IgM anti-GM1 antibodies were raised (1450 AU/1). The table shows electrophysiological features at onset of treatment. 
Table 1 Electrophysiological features of the patient who responded to IVIg therapy

\begin{tabular}{|c|c|c|c|c|c|c|c|}
\hline \multirow[b]{2}{*}{ Nerve } & \multirow[b]{2}{*}{ Stimulation sites } & \multicolumn{3}{|l|}{$C M A P$} & \multirow[b]{2}{*}{$\begin{array}{l}D M L / \text { conduction } \\
\text { velocity }(\mathrm{ms} / \mathrm{m} / \mathrm{s})\end{array}$} & \multicolumn{2}{|l|}{$F$ waves } \\
\hline & & $\begin{array}{l}\text { Amplitude (distal)/ } \\
\text { amplitude reduction } \\
(\mathrm{mV} / \%)\end{array}$ & $\begin{array}{l}\text { Duration } \\
\text { increase (\%) }\end{array}$ & $\begin{array}{l}\text { Area } \\
\text { reduction } \\
(\%)\end{array}$ & & $\begin{array}{l}\text { Minimal } \\
\text { latency (ms) }\end{array}$ & $n$ \\
\hline R Med & W Ep Ax Er & $7.0 / 11-8-10$ & 100 & $179-24$ & $4.4 / 504252$ & 37 & 14 \\
\hline L Med & W Ep Ax Er & $5.5 / 18-110$ & 005 & 2180 & $\overline{3.8} / 51 \overline{57} 58$ & $\overline{33}$ & 16 \\
\hline $\mathrm{R} \operatorname{Med}(\mathrm{F})$ & Ep Ax Er & $9.1 /-130$ & 50 & -160 & $4.1 / 5650$ & 二 & \\
\hline L Med (F) & $\mathrm{Ep} \mathrm{Ax} \mathrm{Er}$ & $4.5 / 00$ & -1015 & 00 & $3.3 / 5373$ & - & \\
\hline R Uln & W Ed Ep Ax Er & $4.2 / 143916$ & 5000 & $11-477$ & $3.3 / 55455853$ & 36 & 17 \\
\hline L Uln & W Ed Ep Ax Er & $4.2 / 108923$ & 0000 & -56420 & $3.3 / 59 \overline{50} 5561$ & $\overline{34}$ & 17 \\
\hline $\mathrm{R} \mathrm{Rad}$ & $\mathrm{Ax} \mathrm{Er}$ & $1.3 / 2$ & 21 & -18 & $2.1 / 50$ & 二 & \\
\hline L Rad & $\mathrm{Ax} \mathrm{Er}$ & $2.1 / 20$ & 0 & 19 & $3.3 / 60$ & - & \\
\hline R Mus & $\mathrm{Ax} \mathrm{Er}$ & $0.3 / 0$ & 0 & 0 & $4.0 / 57$ & - & \\
\hline L Mus & $\mathrm{Ax} \mathrm{Er}$ & $\underline{0.5} / 17$ & -4 & 36 & $3.1 / 63$ & - & \\
\hline R Per & An Fd Fp & $\underline{0.2} / 020$ & 00 & 00 & $\underline{6.6} / \underline{27} 59$ & $\underline{N R}$ & \\
\hline L Per & An Fd Fp & $\overline{0.3} / 00$ & 130 & 00 & $\overline{\overline{7.1}} / \overline{29} 32$ & $\overline{73}$ & 3 \\
\hline R Tib & An $\mathrm{P}$ & $\overline{3.3} / 36$ & 8 & 15 & $\overline{5.3} / \underline{\underline{39}}$ & $\overline{62}$ & 13 \\
\hline L Tib & An $P$ & $3.1 / 32$ & 14 & 34 & $4.9 / \overline{39}$ & $\overline{64}$ & 16 \\
\hline
\end{tabular}

Neurophysiological features of the patient who responded to IVIg treatment. Investigated nerves: R=right; L=left; $M$ ed=median nerve, recording of CMAP from the abductor pollicis brevis muscle; Med $(F)=$ median nerve, recording of CMAP from the flexor carpi radialis muscle; Uln=ulnar nerve; Rad=radial nerve; Mus=musculocutaneous nerve; Per=peroneal nerve; Tib=tibial nerve. Stimulation sites: $\mathrm{W}=$ wrist; $\mathrm{Ed}=5 \mathrm{~cm}$ below the elbow; $\mathrm{Ep}=5 \mathrm{~cm}$ above the elbow; $\mathrm{Ax}=\mathrm{axilla} ; \mathrm{Er}=\mathrm{Erb}$ 's point; $\mathrm{An}=\mathrm{ankle} ; \mathrm{Fd}=5 \mathrm{~cm}$ below the fibular head; $\mathrm{Fp}=5 \mathrm{~cm}$ above the fibular head; $\mathrm{P}=$ popliteal fossa; $\mathrm{CMAP}=$ compound muscle action potential; $\mathrm{DML}=$ distal motor latency; $n=$ number of $F$ waves after 20 stimuli. $\mathrm{NR}=$ no response; - =not done; underlined values are abnormal.

Concentric needle EMG showed spontaneous muscle fibre activity in the left and right biceps brachii, left flexor carpi radialis, left and right first dorsal interosseus, and left and right tibialis anterior muscles. Polyphasic or giant motor unit potentials were seen in the left and right biceps brachii, left flexor carpi radialis, left first dorsal interosseus, and left tibialis anterior muscles. Sensory conduction in the median, ulnar, and sural nerves was normal. A reduced CMAP amplitude was found on distal stimulation of both musculocutaneous nerves and both deep peroneal nerves. No evidence of conduction block was found. In the right peroneal nerve, conduction velocity and $F$ waves were compatible with demyelination, but the CMAP amplitude on distal stimulation was very low $(0.2 \mathrm{mV})$.

\section{Response to IVIg treatment}

Muscle strength improved in three muscle groups after open IVIg treatment: the right foot extensors, the right knee flexors, and the left elbow extensors. The maximum effect was measured two weeks after onset of IVIg treatment and was followed by a gradual decrease in muscle strength over one month. Subjective feelings were in agreement with the findings from myometry: the patient could lift his arms, handle objects easier, and could walk better.

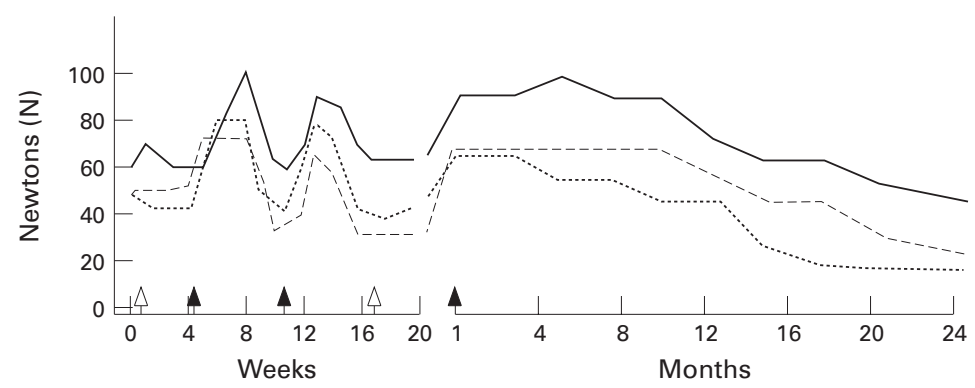

Dynamometric measurements of muscles of the patient who responded to IVIg showing the clearest change in strength in the double blind, placebo controlled study and during weekly maintenance IVIg treatment after the patient had received one full IVIg treatment course $(0.4 \mathrm{~g} / \mathrm{kg}$ for 5 days $) . \ldots .=$ foot extension $;---=R$ knee flexion; $\quad=L$ elbow extension; open triangle $=$ placebo treatment; closed triangle $=I$ IVI treatment $(0.4 \mathrm{~g} / \mathrm{kg}$ for five days).
The beneficial effect of IVIg in the patient was confirmed in the double blind, placebo controlled study: muscle strength improved after IVIg and remained stationary after placebo infusion (figure). The objective improvement in muscle strength paralleled the subjective judgement of the patient.

Subsequently the patient received maintenance IVIg treatment for 24 months. Initially muscle strength could be maintained at the maximum level obtained after a full IVIg treatment course. However, after six months of maintenance IVIg treatment muscle strength gradually declined below pretreatment values (figure). Twenty months after onset of treatment weakness increased and nocturnal artificial ventilation was required. Anti-GM1 antibody titres and electroneurographical follow up (including $\mathrm{F}$ waves) did not change significantly in this period.

\section{Discussion}

In the present study only one of the five patients with lower motor neuron disease associated with anti-GM1 antibodies improved after IVIg treatment. The effect of treatment in this patient was confirmed in a double blind, placebo controlled study to ensure that the improvement in muscle strength was not due to a placebo effect. The response to IVIg treatment in the patient lasted only six months and subsided thereafter despite IVIg maintenance treatment. This is by contrast with our experience with patients with multifocal motor neuropathy, in whom we found further improvement of muscle strength during long term weekly IVIg treatment. ${ }^{8}$ Azulay et al found improvement of muscle strength after IVIg treatment in two out of seven patients with lower motor neuron disease associated with raised IgG anti-GM1 antibodies, but long term follow up of these patients was not reported.

As the responding patient did not have definite or possible conduction block on extensive electrophysiological examination a diagnosis of multifocal motor neuropathy could not be made. It has recently been suggested, however, 
that patients with electrophysiological features of demyelination other than conduction block might also have multifocal motor neuropathy. ${ }^{20}$ In our patient the conduction velocity and $F$ waves were in the demyelinating range in only one nerve. However, in this nerve the CMAP amplitude was too low to be conclusive for demyelination.

Although two previous studies did not find an effect on muscle strength with prednisone or cyclophosphamide treatment, ${ }^{21}{ }^{22}$ Pestronk et $a l^{13}$ reported four patients with lower motor neuron disease with raised IgM anti-GM1 antibodies who improved after monthly plasma exchange and intravenous cyclophosphamide treatment. The most prominent improvement occurred six to 24 months after the onset of treatment and would have been absent if observations had been limited to shorter times. Therefore it is possible that in our open study one IVIg treatment, which was shown to be effective in Guillain-Barré syndrome, chronic inflammatory demylinating polyneuropathy, and multifocal motor neuropathy in previous studies, ${ }^{72}$ was not sufficient to measure improvement in patients with lower motor neuron disease associated with anti-GM1 antibodies. However, the patient who initially responded to IVIg in our study received IVIg for 24 months and even further deteriorated during this treatment.

We conclude that the presence of raised anti-GM1 antibodies does not identify a subgroup of patients with lower motor neuron disease who respond to IVIg treatment and although some patients with lower motor neuron disease may initially respond, IVIg therapy does not seem to be sufficient as long term treatment.

This work was supported by the Dutch Organization for Scientific Research.

1 Wokke JHJ. Riluzole. Lancet 1996;348:795-9.

2 Wokke JHJ. Diseases that masquerade as motor neuron disease. Lancet 1996;347:1347-8.
3 Nobile-Orazio E. Multifocal motor neuropathy. 7 Neurol Neurosurg Psychiatry 1996;60:599-603.

4 Willison HJ. Antiglycolipid antibodies in peripheral neuropathy: fact or fiction. $\mathcal{F}$ Neurol Neurosurg Psychiatry 1994;57:1303-7.

5 Pestronk A, Cornblath DR, Ilyas AA, et al. A treatable multifocal motor neuropathy with antibodies to the GM1 ganglioside. Ann Neurol 1988;24:73-8.

6 Nobile-Orazio E, Meucci N, Barbieri S, Carpo M, Scarlato G. High-dose intravenous immunoglobulin therapy in multifocal motor neuropathy. Neurology 1993;43:537-44.

7 Van den Berg LH, Kerkhoff H, Oey PL, et al. Treatment of multifocal motor neuropathy with high-dose intravenous immunoglobulins: a double blind, placebo controlled study. F Neurol Neurosurg Psychiatry 1995;59:248-52.

8 Van den Berg LH, Franssen H, Wokke JHJ. Improvement of multifocal motor neuropathy during long-term weekly treatment with human immunoglobulin. Neurology 1995; treatment

9 Azulay JP, Blin O, Pouget J, et al. Intravenous immunoglobuin treatment in patients with motor neuron syndromes associated with anti-GM1 antibodies: a double-blind, placebo-controlled study. Neurology 1994;44:429-32.

10 Sadiq SA, Thomas FP, Kilidireas K, et al. the spectrum of neurologic disease associated with anti-GM1 antibodies. Neurology 1990;40:1067-72.

11 Van den Berg LH, Marrink J, de Jager AEJ, et al. Anti-GM1 antibodies in patients with Guillain-Barré syndrome. $\mathcal{F}$ Neurol Neurosurg Psychiatry 1992;55:8-11.

12 Van der Meche FGA, Van Doorn PA. Guillain-Barré syndrome and CIDP: immune mechanisms and update on current therapies. Ann Neurol 1995;37(suppl 1):S14-31.

13 Pestronk A, Lopate A, Kornberg AJ, et al. Distal lower motor neuron syndrome with high-titer serum IgM anti-GM1 antibodies: improvement following immunotherapy with monthly plasma exchange and intravenous cyclophosphamide. Neurology 1994;44:2027-31.

14 Kimura J. Electrodiagnosis in diseases of nerve and muscle: principles and practice, 2nd ed. Philadelphia: FA Davis, 1989:141-4.

15 Franssen $\mathrm{H}$, Wieneke GH Nerve conduction and temperature: necessary warming time. Muscle Nerve 1994; 17:336-44.

16 Rhee EK, England JD, Sumner AJ. A computer simulation of conduction block: effects produced by actual block versus interphase cancellation. Ann Neurol 1990;28:146-56.

17 Albers JW, Donofrio PD, McGonagle TK. Sequential electrodiagnostic abnormalities in acute inflammatory demyelinating polyradiculoneuropathy. Muscle Nerve 1985;8:52839.

18 Lange DJ, Trojaborg W, Latov N, et al. multifocal motor neuropathy with conduction block: is it a distinct clinical entity? Neurology 1992;42:497-505.

19 Ad hoc subcommittee of the AAN. Research criteria for diagnosis of CIDP. Neurology 1991;41:617-8.

20 Pakian A, Parry GJ. Multifocal motor neuropathy without evidence of conduction block. Neurology 1996;46:A234-5.

21 Tan E, Lynn J, Amato AA, et al. Immunosuppressive treatment of motor neuron syndromes: attempts to distinguish a treatable disorder. Arch Neurol 1994;51:194-200.

22 Tsai C, Lin K, Liao KK, et al. Immunosuppressive treatment in lower motor neuron syndrome with autoantibodies against GM1 ganglioside. Eur Neurol 1993;33:446-9. 